\title{
Development of key quality indicators for appropriate antibiotic use in the Republic of Korea: results of a modified Delphi survey
}

Bongyoung Kim ${ }^{1 \dagger}$, Myung Jin Lee ${ }^{2 \dagger}$, Se Yoon Park ${ }^{3^{*}+}$ (]) Song Mi Moon ${ }^{4^{*}+}$, Kyoung-Ho Song ${ }^{5}$, Tae Hyong Kim³, Eu Suk Kim ${ }^{5}$, Hong Bin Kim ${ }^{5}$ and Korea Study Group for Antimicrobial Stewardship (KOSGAP)

\begin{abstract}
Background: An effective antibiotic stewardship program relies on the measurement of appropriate antibiotic use, on which there is a lack of consensus. We aimed to develop a set of key quality indicators (Qls) for nationwide point surveillance in the Republic of Korea.

Methods: A systematic literature search of PubMed, EMBASE, and Cochrane Library (publications until 20th November 2019) was conducted. Potential key Qls were retrieved from the search and then evaluated by a multidisciplinary expert panel using a RAND-modified Delphi procedure comprising two online surveys and a face-to-face meeting.

Results: The 23 potential key Qls identified from 21 studies were submitted to 25 multidisciplinary expert panels, and 17 key Qls were retained, with a high level of agreement (13 Qls for inpatients, 7 for outpatients, and 3 for surgical prophylaxis). After adding up the importance score and applicability, six key Qls [6 Qls (Q 1-6) for inpatients and 3 (Q 1,2 , and 5) for outpatients] were selected. (1) Prescribe empirical antibiotic therapy according to guideline, (2) change empirical antibiotics to pathogen-directed therapy, (3) obtain culture samples from suspected infection sites, (4) obtain two blood cultures, (5) adapt antibiotic dosage to renal function, and (6) document antibiotic plan. In surgical prophylaxis, the QIs to prescribe antibiotics according to the guideline and initiate antibiotic therapy $1 \mathrm{~h}$ before incision were selected.
\end{abstract}

Conclusions: We identified key Qls to measure the appropriateness of antibiotic therapy to identify targets for improvement and to evaluate the effects of antibiotic stewardship intervention.

Keywords: Quality indicator, Anti-bacterial agents, Antibiotic prophylaxis, Bacterial infection

*Correspondence: livinwill2@gmail.com; moon7796@hanmail.net ${ }^{\dagger}$ Bongyoung Kim and Myung Jin Lee have contributed equally as first authors

${ }^{\dagger}$ Se Yoon Park and Song Mi Moon have contributed equally as corresponding authors

${ }^{3}$ Division of Infectious Diseases, Department of Internal Medicine, Soonchunhyang University Seoul Hospital, Soonchunhyang University College of Medicine, 59, Daesagwan-ro, Yongsan-gu, Seoul 140-743, Republic of Korea

${ }^{4}$ Department of Internal Medicine, Hallym University Sacred Heart Hospital, Anyang, Republic of Korea

Full list of author information is available at the end of the article

\section{Background}

The increased use of broad-spectrum antibiotics is closely related to antimicrobial resistance $[1,2]$. Therefore, a need to assess appropriate antibiotic use has been emphasized [3] and includes various steps, such as diagnosis, empirical therapy, dosing, de-escalation, and therapy duration [4]. Moreover, the appropriateness can change with the definition of appropriate antibiotic use [5]. Therefore, researchers have attempted to evaluate antibiotic use's appropriateness with standard criteria by using quality indicators (QIs) developed through expert consensus. In 2018, the European Surveillance 
of Antimicrobial Consumption group developed QIs to evaluate appropriate antibiotic use in inpatients and outpatients in Europe [6, 7]. In Australia, surveillance of appropriate antibiotic use has been conducted since 2011. Key indicators have been developed [8]; results of appropriate antibiotic use have been generated through national surveillance every year using the key indicators. Nonetheless, it is important to evaluate appropriate antibiotic use with standard criteria, which can then be directly compared between medical institutions and provide feedback to generate suggestions to improve the goals of national antibiotic stewardship. The QIs are diverse, and some indicators are difficult to apply and; therefore, it is necessary to select appropriate QIs through consensus on the antibiotic prescription situation (location), infectious disease, and the type of antibiotics.

In the Republic of Korea (ROK), point surveillance of appropriate antibiotic use was conducted for 20 medical institutions in 2018, and the appropriateness was determined based on expert opinion [9]. This experience generated a need to evaluate appropriate antibiotic use based on specific standards. This study aimed to select potential QIs for appropriate antibiotic use through a systematic literature search and determine the key QIs necessary and important in the ROK through expert consensus. In addition, the developed key QIs will be used in future nationwide point surveillance of appropriate antibiotic use.

\section{Methods}

We used a four-step RAND-modified Delphi method to develop a set of QIs to measure the appropriateness of antimicrobial use in adult and hospitalized pediatric patients, outpatients, or patients receiving surgical prophylaxis [10, 11]. Figure 1 presents an overview of the RAND-modified Delphi procedure, which included a comprehensive literature review to develop a list of candidate key QIs, two rounds of an online survey, and a face-to-face meeting with the panelists. The consensus procedure combined the individual opinions of multidisciplinary expert panels. All the panel members consented to participate in the study and were aware that their answers would be used for research.

\section{Systematic search for generating key Qls}

We performed a systematic search using a protocol designed by two independent medical librarians (D.W.S and M.L). We screened the literature using databases of PubMed, EMBASE, and Cochrane Library for papers published up to 20th November 2019. The search strategy is shown in Additioinal file 1: Fig. S1 and was directed towards identifying evidence-based QIs for antibiotic use (e.g., literature review or evidence-based guideline). Papers written in English and discussed using systemically administered antibiotic drugs in inpatients, outpatients, and surgical prophylaxis were included, except for case reports. This study aimed to develop key QIs useful to assess the antibiotic use appropriateness in treating all bacterial infections and surgical prophylaxis. Therefore, we excluded antiviral, antifungal, antiparasitic, or antituberculosis drugs. Using EndNote software (version X7.1, 2020 Clarivate), two researchers (B.K. and S.Y.P.) independently examined all titles and abstracts to select papers that described QIs. Any disagreement on the inclusion or exclusion of studies was resolved through discussion with a third author (S.M.). If no abstract was available or there was a lack of information for the eligibility assessment, papers were selected for full-text screening. The inclusion/exclusion criteria evaluation for full-text screening was performed by two researchers (B.K. and S.Y.P.).

\section{Selection of potential key Qls}

Data on potential QIs were extracted by four researchers (B.K., M.J.L., S.Y.P., and S.M.). The QIs were excluded if they were: not concerned with antibiotic use for a specific group of patients, non-normative, or developed for each institution and not for patients. The extracted QIs were then clustered into different non-overlapping logical themes based on the definition of responsible use. When a QI could be allocated to more than one theme, the predominant theme was chosen based on consensus between two authors (B.K. and S.Y.P.). Duplicates were removed, and the QIs were rephrased as a recommendation. The clustering, aggregating, and rephrasing steps were undertaken consensually among four authors (B.K., M.J.L., S.Y.P., and S.M.).

\section{First online survey}

We emailed invitations to different specialists for their participation in 25 expert panels. The panel of doctors comprised experts working at university-affiliated hospitals in the ROK. During panel selection, we aimed to select experts representative and responsible for antibiotic prescriptions. The panel comprised infectious disease specialists $(n=13)$, laboratory medicine doctors $(n=3)$, pediatric infectious diseases specialists $(n=2)$, urologists $(\mathrm{n}=2)$, otorhinolaryngologist $(\mathrm{n}=1)$, gastroenterologist $(n=1)$, pulmonologist $(n=1)$, general surgeon $(n=1)$, and researchers of the National Evidence-Based Healthcare Collaborating Agency $(n=1)$ (Additioinal file 3: Table S2). To rate the degree to which the potential QI described appropriate antibiotic use, a Likert scale, ranging from 1 ('definetly inappropriate care') to 7 ('definitely appropriate care'), was used. The 
Step 1. Electronic database search result $(n=4,293)$

Pubmed $(n=1,415)$, Cochrane $(n=141)$, Embase $(n=2,737)$

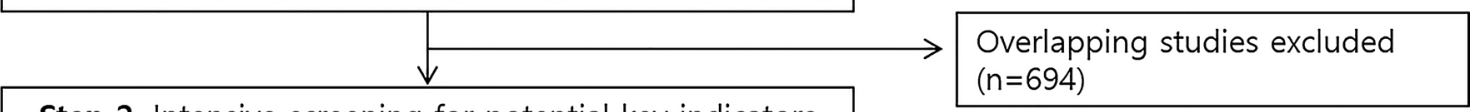

Step 2. Intensive screening for potential key indicators by three reviewers

Total selected studies $(n=21)$

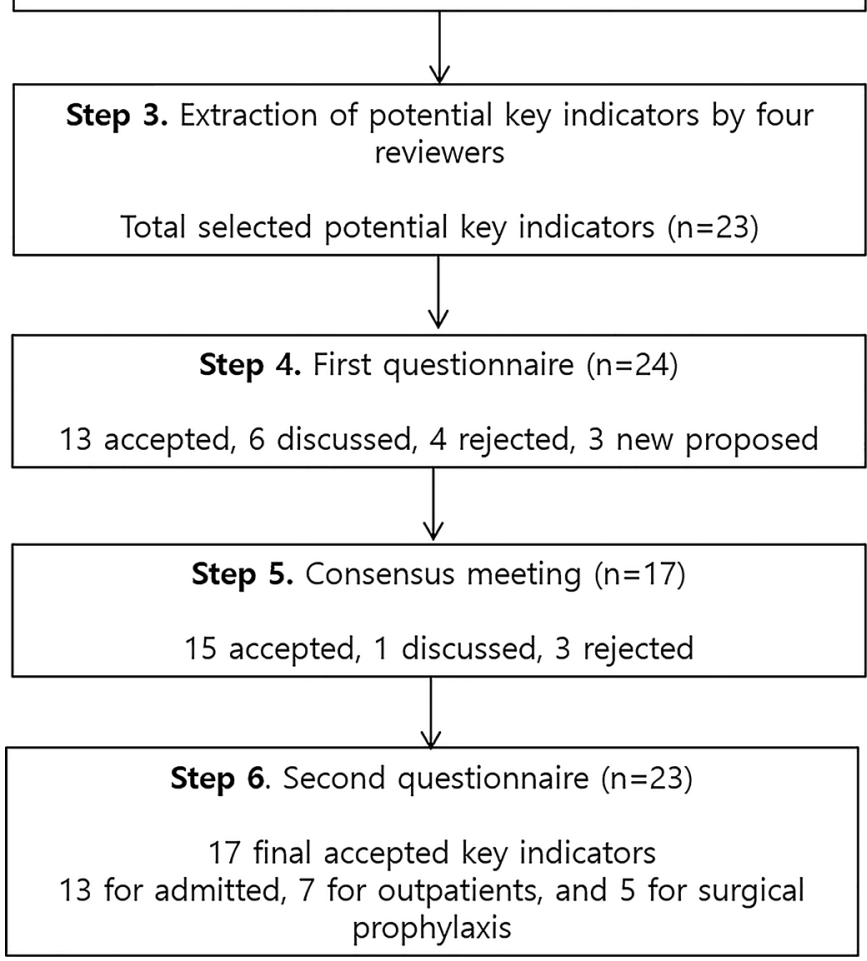

Fig. 1 Flowchart of the study procedures

panelists could rephrase the potential indicator and could even add new items. A consensus was defined as the case wherein $\geq 70 \%$ of the scores were in the top quartile (scores 6 and 7); similarly, scores with $<70 \%$ agreement were defined as disagreement. QIs with a median score of 6 or 7 were accepted if there was agreement. If there was disagreement and the median score was $\leq 5$, the QI was rejected. Thus, QIs with a median score of 6 and 7 with disagreement were discussed during the expert panel meeting. In addition, we graded each QI using a Likert scale score, ranging from 1 to 7 , as relevant to inpatient care, outpatient care, or surgical prophylaxis. If the score was 6 or 7, we considered it to be an appropriate QI.

\section{Expert panel meeting}

All panel members were invited to a face-to-face panel meeting. Before the meeting, all participants received a personal feedback report with the results of the first online questionnaire. The agenda of the panel meeting was to present the results of the first round of the survey and to discuss the QIs with a median score of 6 or 7 that had inadequate consensus. These QIs were accepted if at least $70 \%$ of the experts concurred. In addition, newly added potential QIs were discussed, and the accepted QIs with comments from the experts were rephrased base on consensus.

\section{Second online survey}

A second questionnaire that included all the selected and rephrased QIs were sent with a personal feedback report (providing the results of the previous two steps of the consensus procedure) to all participating panelists. The panelists were asked to select from the following three answers: 'agree,' 'disagree,' and 'cannot assess.' The 
rephrased indicators were accepted if at least $70 \%$ of the experts agreed with the new formulation. Furthermore, we asked the panelists to consider the importance of potential QIs that could be used as key indicators for antibiotic use in the ROK, with a Likert scale score from 1 'less important' to 5 'highest importance.' If the QIs could be evaluated in point surveillance, we considered them applicable. This was finally confirmed by four researchers (B.K., M.J.L., S.Y.P., and S.M.). We excluded QIs with durations in which appropriateness was difficult to evaluate by point surveillance.

\section{Results}

\section{Literature search and selection of potential key QIs}

The systematic literature search identified 4293 articles, of which only 21 articles were finally selected after screening against the inclusion/exclusion criteria (Additioinal file 2: Table S1) [6, 7, 12-29]. From these 21 articles, we initially derived 192 QIs. After duplications were removed and the quantitative or institution-level QIs were excluded, 23 QIs were finally included (Table 1). This list of 23 QIs was presented to the panelists in the first round of the consensus procedure.

\section{First online survey}

The first online round of the survey was conducted from 27th December 2019 to 8th January 2020. In total, 24 (96\%, 24/25) panelists responded to the first online questionnaire. Among the 23 potential QIs, 13 QIs (57\%) were accepted, $6(26 \%)$ were discussed, and $4(17 \%)$ were rejected (Table 2). Three additional QIs were suggested from the results of the online survey: (1) dose adjustment of surgical antibiotic prophylaxis according to body weight, (2) Percentage of blood cultures among those who received systemic antibiotic use, and (3) empirical antibiotic therapy should be prescribed in communityacquired or healthcare-associated infections. The application of QIs was appropriate in hospitalized patients $(\mathrm{n}=17)$, outpatients $(\mathrm{n}=9)$, and surgical prophylaxis $(\mathrm{n}=6)$.

Table 1 Final lists of potential key quality indicators

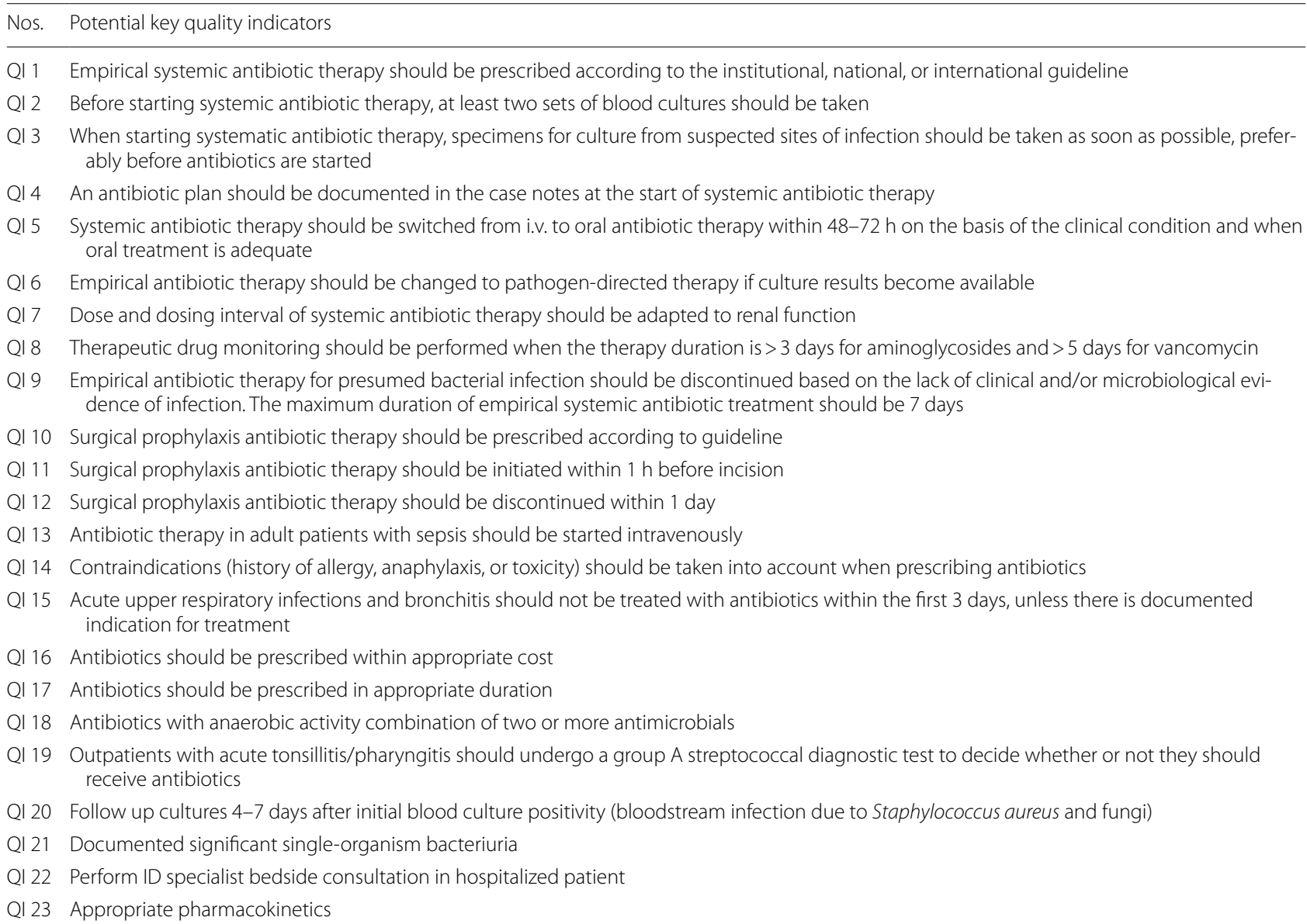




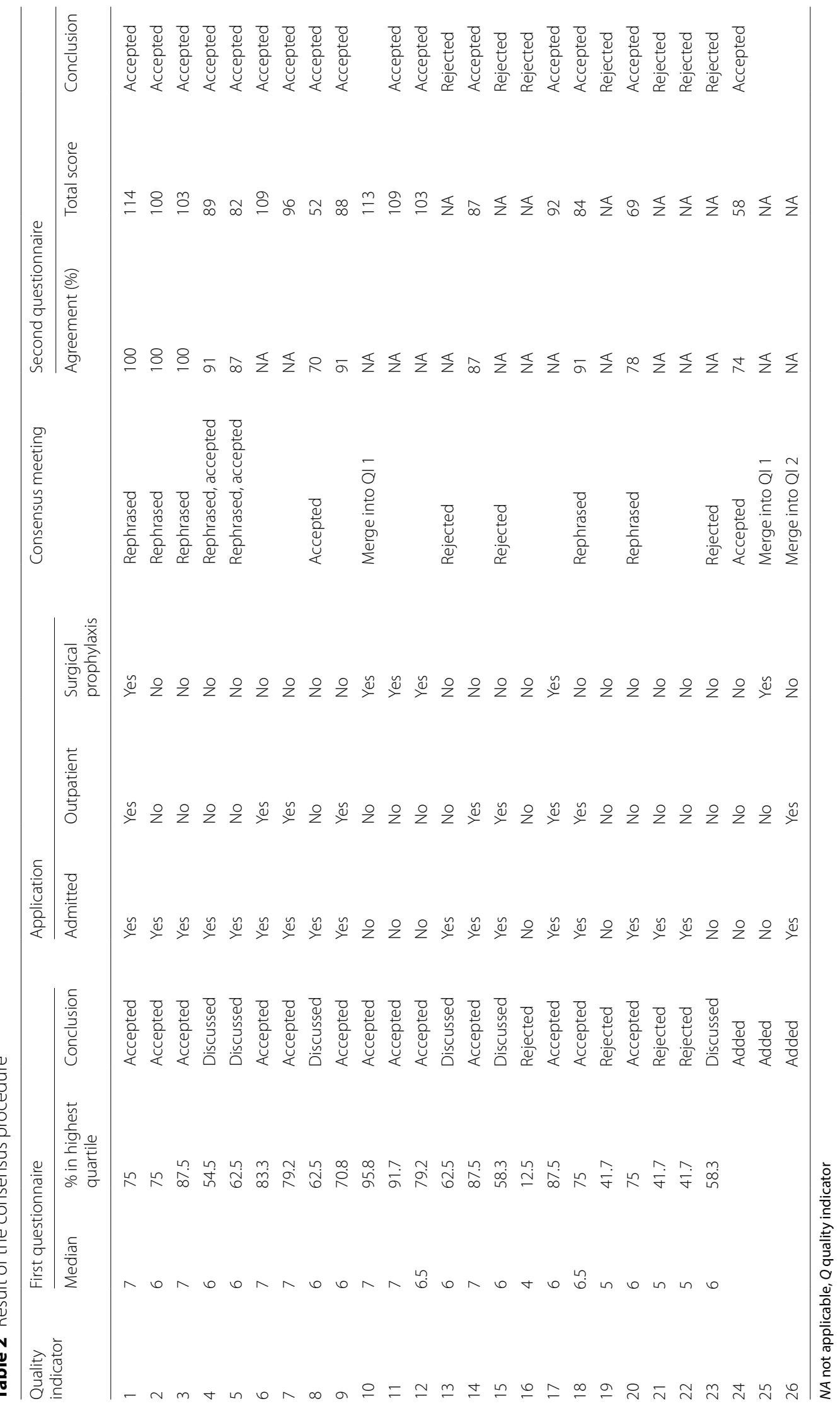




\section{Expert panel meeting}

The face-to-face panel meeting was held on 10th January 2020 , and $16(64 \%, 16 / 25)$ panelists participated in this meeting. Among the six potential QIs from the first online questionnaire round discussed, three were conclusively accepted, and three were rejected. QI 10 was merged with QI 1. Among the three suggested QIs from the first survey, one QI (dose adjustment of surgical antibiotic prophylaxis according to body weight) was accepted. The other two QIs (percentage of blood culture among those with systemic antibiotic use and empirical antibiotic therapy should be prescribed in community-acquired or healthcare-associated infections) were merged with QI 1 and QI 2, respectively (Table 2).

\section{Second online survey}

A total of 17 potential QIs (16 pre-existing QIs, and one new QI was added) were included in the second online questionnaire. Twenty-three $(92 \%, 23 / 25)$ panelists participated in this survey. All potential QIs were accepted (Table 2). The ranking of the final selected key QIs and applicability in the point surveillance study is shown in Table 3 (inpatients and out-patients) and Table 4 (surgical prophylaxis). Based on the sum of the importance score and applicability, six key QIs for hospitalized patients and three (following number 1-3) for outpatients were finally selected. (1) Prescribe empirical antibiotic therapy according to the guideline, (2) change empirical antibiotic to pathogen-directed therapy, (3) obtain culture samples from suspected sites of infection, (4) take two blood cultures, (5) adapt antibiotic dosage to renal function, and (6) document the antibiotic plan. Concerning surgical prophylaxis, the recommendations to prescribe according to the guideline and initiate antibiotics $1 \mathrm{~h}$ before the incision were finally selected.

Table 3 Ranking of final selected key quality indicators for admitted and outpatients and applicability in point surveillance study

\begin{tabular}{|c|c|c|c|c|c|c|}
\hline \multirow[t]{2}{*}{ Quality indicators } & \multicolumn{3}{|c|}{ Inpatients } & \multicolumn{3}{|c|}{ Outpatients } \\
\hline & Ranking & Total score & Applicability & Ranking & Total score & Applicability \\
\hline $\begin{array}{l}\text { QI 1. Empirical systemic antibiotic therapy should be prescribed } \\
\text { according to the institutional, national, or international guideline }\end{array}$ & 1 & 114 & Yes & 1 & 114 & Yes \\
\hline $\begin{array}{l}\text { QI 6. Empirical antibiotic therapy should be changed to pathogen- } \\
\text { directed therapy if culture results become available }\end{array}$ & 2 & 109 & Yes & 2 & 109 & Yes \\
\hline $\begin{array}{l}\text { Q1 3. When starting systematic antibiotic therapy, specimens for } \\
\text { culture from suspected sites of infection should be taken as soon as } \\
\text { possible, preferably before antibiotics are started }\end{array}$ & 3 & 103 & Yes & & & \\
\hline $\begin{array}{l}\text { QI 2. Before starting systemic antibiotic therapy, at least two sets of } \\
\text { blood cultures should be taken }\end{array}$ & 4 & 100 & Yes & & & \\
\hline $\begin{array}{l}\text { Q1 7. Dose and dosing interval of systemic antibiotic therapy should be } \\
\text { adapted to renal function }\end{array}$ & 5 & 96 & Yes & 3 & 96 & Yes \\
\hline QI 17. Antibiotics should be prescribed in appropriate duration & 6 & 92 & No & 4 & 92 & No \\
\hline $\begin{array}{l}\text { QI 4. An antibiotic plan should be documented in the case notes at } \\
\text { the start of systemic antibiotic therapy }\end{array}$ & 7 & 89 & Yes & & & \\
\hline $\begin{array}{l}\text { Q1 9. Empirical antibiotic therapy for presumed bacterial infection } \\
\text { should be discontinued based on the lack of clinical and/or micro- } \\
\text { biological evidence of infection. The maximum duration of empirical } \\
\text { systemic antibiotic treatment should be } 7 \text { days }\end{array}$ & 8 & 88 & No & 5 & 88 & No \\
\hline $\begin{array}{l}\text { QI 14. Contraindications (history of allergy, anaphylaxis, or toxicity) } \\
\text { should be taken into account when prescribing antibiotics }\end{array}$ & 9 & 87 & No & 6 & 87 & No \\
\hline $\begin{array}{l}\text { Ql 18. Antibiotics with anaerobic activity combination of two or more } \\
\text { antimicrobials }\end{array}$ & 10 & 84 & No & & & \\
\hline $\begin{array}{l}\text { Q } 5 . \text { Systemic antibiotic therapy should be switched from i.v. to oral } \\
\text { antibiotic therapy within } 48-72 \mathrm{~h} \text { on the basis of the clinical condi- } \\
\text { tion and when oral treatment is adequate }\end{array}$ & 11 & 82 & No & & & \\
\hline $\begin{array}{l}\text { QI 20. Follow up cultures 4-7 days after initial blood culture positivity } \\
\text { (bloodstream infection due to Staphylococcus aureus and fungi) }\end{array}$ & 12 & 69 & No & & & \\
\hline $\begin{array}{l}\text { Q } 8 \text {. Therapeutic drug monitoring should be performed when the } \\
\text { therapy duration is }>3 \text { days for aminoglycosides and }>5 \text { days for } \\
\text { vancomycin }\end{array}$ & 13 & 52 & No & 7 & 84 & No \\
\hline
\end{tabular}

Q/ quality indicator 
Table 4 Ranking of final selected key quality indicators for surgical prophylaxis and applicability in point surveillance study

\begin{tabular}{llcc}
\hline Ranking & Quality indicators & Total score & Applicability \\
\hline 1 & Ql 10. Surgical prophylaxis antibiotic therapy should be prescribed according to guideline & 113 & Yes \\
2 & Ql 11. Surgical prophylaxis antibiotic therapy should be initiated within 1 h before incision & 109 & Yes \\
3 & Ql 12. Surgical prophylaxis antibiotic therapy should be discontinued within 1 day & 103 & No \\
4 & Ql 17. Antibiotics should be prescribed in appropriate duration & 92 & No \\
5 & Ql 24. Dose of surgical prophylaxis antibiotics should be adjusted according to body weight & 58 & No \\
\hline
\end{tabular}

Q/ quality indicator

\section{Discussion}

We selected key QIs from among the potential QIs extracted in a systematic literature search followed by a RAND-modified Delphi survey procedure. These key QIs are intended to be universally applicable, regardless of the type of infectious disease, location, or situation of antibiotic use. This study is practical in that the key QIs can be applied in all antibiotic use situations, including inpatients, outpatients, and surgical prophylaxis, and were selected through an expert panel consensus with ranking for importance and consideration of applicability in point surveillance. The selected key QIs for hospitalized patients and outpatients should be applied in a point surveillance study for antibiotic appropriateness in 75 hospitals in the ROK in 2020.

Finally, selected Key QIs were included in several steps of antibiotic prescription, such as diagnosis (QI 2, 3, and 4), empiric therapy (QI 1), dosing (QI 7), and definite therapy (QI 6). QIs related to therapy duration were not selected, although it got a considerably high score on the importance scale among experts. Therapy duration is increasingly an important focus area for antimicrobial stewardship activity. However, expected applicability was below $10 \%$ based on previous studies $[9,13,22]$. Therefore, we excluded these QIs in the final selection. If point surveillance or applicability is not considered, the QIs selected in the second online survey can be used by an antibiotic stewardship team or a researcher, depending on the surveillance purpose.

Assessing antibiotic use's appropriateness can be divided mainly into evaluating the entire antibiotic options and evaluating certain antibiotics or diseases [30]. In addition, the scope of assessment varies greatly depending on the study, from empirical antibiotics to therapeutic antibiotics, dosing, narrow-spectrum antibiotics, duration of treatment, and presence of medical records [4]. Moreover, the methods of evaluation can be divided into judgments based on guidelines, judgment by experts, and judgments using QIs [5]. Therefore, the scope of evaluation and the criteria for assessing appropriate antibiotic use should be determined by researchers according to the surveillance purpose. Because of the increasing need for antibiotic stewardship, national surveillance of appropriate antibiotic use commenced in 2018 in the ROK. To establish a national target of antibiotic stewardship, we included the entire gamut of antibiotic use, regardless of infectious disease type.

To date, the most widely used QIs are 11 indicators that were selected by van den Bosch et al. [14] in the Netherlands. These QIs were selected from 1574 studies through a literature review. The RAND-modified Delphi procedure was used to propose the final 11 QIs; of these, nine were proposed at the patient level and two at the institutional level [14]. Those 11 QIs were applied to 1890 inpatients in 22 hospitals in the Netherlands in 2011 and 2012. The applicability of three QIs (therapeutic drug monitoring, adapting antibiotics to renal function, discontinuation of empirical therapy in case of a lack of clinical and/or microbiological evidence of infection) were less than $10 \%$ [13]. In this study, the nine potential key QIs of patient-level by van den Bosch et al. were merged and finally selected after obtaining consensus. Considering their applicability in the point surveillance study, we finally excluded key QIs on duration, such as "appropriate duration" and "discontinue antibiotic therapy if an infection is not confirmed." The "adapted dose and dosing interval of antibiotics to renal function" QI was expected to have low applicability. However, it was finally accepted because this key QI can be evaluated by point surveillance, and the panel considered it important.

The advantage of QIs over expert judgment is that the evaluation results of each QI show the areas that need improvement. In addition, comparisons with other medical institutions show whether the specified institution needs improvement. Another advantage is that the same criteria can be applied every year to determine whether one's institution has improved. In Australia, four key QIs (documentation of indication, documentation of review or stop date, surgical prophylaxis for $>24 \mathrm{~h}$. and noncompliance with guidelines) and inappropriate antibiotic prescription rates are presented annually along with the target levels [8]. Based on the collected data, the researchers could identify priority areas for targeted quality improvement initiatives directed toward 
antimicrobial prescribing patterns. In comparison, judgments may differ among experts, and it is difficult to ascertain the aspects required for improvement. Thus, efforts to select the key QIs agreed upon by each country or institution are a necessary task in the future.

A limitation of this study is that members of the expert panel were only Koreans, and none was with pharmacy expertise. The QIs would be difficult to apply in other countries with different medical environments from the ROK. Pharmacy expertise was suggested as a core element of hospital antibiotic stewardship programs; however, the current antimicrobial stewardship program is mainly performed by infectious diseases specialists in the ROK [31, 32]. Second, the literature search scope was restricted to English-only papers and limited to only a literature database, without a website search. Therefore, we may have missed important papers in other languages. However, English is considered a major global medium in the scientific literature directed toward a global audience.

\section{Conclusions}

In this study, key QIs that can be applied to hospitalized patients, outpatients, and surgical prophylaxis were selected in the ROK. Not only will these key QIs be applied in national point surveillance initiatives in the ROK in 2020, but also other users will be able to use the QIs for antibiotic stewardship programs or in educational materials for appropriate antibiotic use. Evaluators will be able to prioritize antibiotic use based on the important indicators identified in this study. It is expected that the application of key QIs will complement and apply the advantages and limitations of the evaluation indicators experienced by the actual evaluators and researchers.

\section{Abbreviations}

\section{QI: Quality indicators; ROK: Republic of Korea.Supplementary Information}

The online version contains supplementary material available at https://doi. org/10.1186/s13756-021-00913-y.

Additional file 1. Figure: Search strategy for the literature review.

Additional file 2. Table 1: Final list of 21 articles selected after a systematic literature search.

Additional file 3. Table 2: Title and affiliations of expert panels.

\section{Acknowledgements}

The authors are grateful to all panel members who participated in the consensus procedures. These include Yoon Soo Park, Joon Young Song, Mi Suk Lee, Hyung-Ha Chang, Su-Mi Choi, Ji Young Park, Hyunju Lee, Ki-Ho Hong, Jeong Su Park, Jae Wook Kim, Jaihwan Kim, Seung-Ju Lee, U-Syn Ha, Hyuk Min Lee, Jee Youn Oh, Kil Yeon Lee, and Dong-A Park. The authors also thank Misoon Lim and Dong Won Shin (Medical Library, Soonchunhyang University Seoul Hospital), who assisted with the search strategy.

\section{Authors' contributions}

ESK and HBK developed the study design. BK, MJL, SYP, and SMM were responsible for data collection. THK, K-HS, ESK, and HBK assisted data interpretation. BK, MJL, SYP, and SMM performed the literature search. BK and SYP wrote the first draft of the paper. All authors have critically read and commented on draft versions of the report and approved the final manuscript. All authors read and approved the final manuscript.

\section{Funding}

This work was supported by a research program funded by the Korea Centers for Disease Control and Prevention (Grant No. 2019E280400). The funders had no role in the study design, data collection and analysis, preparation of the manuscript, or the decision to publish.

\section{Availability of data and materials}

The datasets used and/or analyzed during the current study are available from the corresponding author on reasonable request.

\section{Ethics approval and consent to participate}

This work was approved by the Institutional Review Board of each hospital (IRB No. 2019-12-016).

\section{Consent for publication}

Not applicable.

\section{Competing interests}

The authors have no potential conflicts of interest to declare.

\section{Author details}

${ }^{1}$ Department of Internal Medicine, Hanyang University College of Medicine, Seoul, Republic of Korea. ${ }^{2}$ Department of Internal Medicine, Inje University Sanggye Paik Hospital, Seoul, Republic of Korea. ${ }^{3}$ Division of Infectious Diseases, Department of Internal Medicine, Soonchunhyang University Seoul Hospital, Soonchunhyang University College of Medicine, 59, Daesagwan-ro, Yongsan-gu, Seoul 140-743, Republic of Korea. ${ }^{4}$ Department of Internal Medicine, Hallym University Sacred Heart Hospital, Anyang, Republic of Korea. ${ }^{5}$ Division of Infectious Diseases, Department of Internal Medicine, Seoul National University Bundang Hospital, Seoul National University College of Medicine, Seongnam, Republic of Korea.

Received: 17 November 2020 Accepted: 19 February 2021

Published online: 06 March 2021

\section{References}

1. Kim B, Kim Y, Hwang H, Kim J, Kim SW, Bae IG, et al. Trends and correlation between antibiotic usage and resistance pattern among hospitalized patients at university hospitals in Korea, 2004 to 2012: a nationwide multicenter study. Medicine (Baltimore). 2018;97:e13719.

2. Kim YA, Park YS, YoukT, Lee H, Lee K. Changes in antimicrobial usage patterns in Korea: 12-year analysis based on Database of the National Health Insurance Service-National Sample Cohort. Sci Rep. 2018;8:12210.

3. Barlam TF, Cosgrove SE, Abbo LM, MacDougall C, Schuetz AN, Septimus EJ, et al. Implementing an Antibiotic Stewardship Program: guidelines by the Infectious Diseases Society of America and the Society for Healthcare Epidemiology of America. Clin Infect Dis. 2016;62:e51-77.

4. Spivak ES, Cosgrove SE, Srinivasan A. Measuring appropriate antimicrobial use: attempts at opening the black box. Clin Infect Dis. 2016;63:1639-44.

5. DePestel DD, Eiland EH 3rd, Lusardi K, Destache CJ, Mercier RC, McDaneld PM, et al. Assessing appropriateness of antimicrobial therapy: in the eye of the interpreter. Clin Infect Dis. 2014;59(Suppl 3):S154-61.

6. Monnier AA, Schouten J, Le Marechal M, Tebano G, Pulcini C, Stanic Benic $M$, et al. Quality indicators for responsible antibiotic use in the inpatient setting: a systematic review followed by an international multidisciplinary consensus procedure. J Antimicrob Chemother. 2018;73(Suppl 6):vi30-9.

7. Le Maréchal M, Tebano G, Monnier AA, Adriaenssens N, Gyssens IC, Huttner B, et al. Quality indicators assessing antibiotic use in the outpatient setting: a systematic review followed by an international multidisciplinary consensus procedure. J Antimicrob Chemother. 2018;73(Suppl 6):vi40-9. 
8. Australian Commission on Safety and Quality in Health Care. Antimicrobial prescribing practice in Australian hospitals: results of the 2017 Hospital National Antimicrobial Prescribing Survey. Available from: https ://www.safetyandquality.gov.au/wp-content/uploads/2018/11/2017Hospital-NAPS.pdf.

9. Park SY. Use of quality indicators and expert opinion for evaluation of antimicrobial appropriateness Chungcheongnam-do. Soonchunhyang University; 2019.

10. Campbell SM, Braspenning J, Hutchinson A, Marshall M. Research methods used in developing and applying quality indicators in primary care. Qual Saf Health Care. 2002;11:358-64.

11. Fitch K, Bernstein SJ, Aguilar MD, Burnand B, LaCalle JR, Lázaro P. Loo Mirjam, et al. The Rand/UCLA Appropriateness Method User's Manual; 2001.

12. van der Velden AW, Roukens M, van de Garde E, Lourens M, Natsch S. SWAB's working group on surveillance of antimicrobial use. Usefulness of quality indicators for antibiotic use: case study for the Netherlands. Int J Qual Health Care. 2016;26:838-42.

13. van den Bosch CM, Hulscher ME, Natsch S, Wille J, Prins JM, Geerlings SE. Applicability of generic quality indicators for appropriate antibiotic use in daily hospital practice: a cross-sectional point-prevalence multicenter study. Clin Microbiol Infect. 2016;22:888.e1-888.e9.

14. van den Bosch CM, Geerlings SE, Natsch S, Prins JM, Hulscher ME. Quality indicators to measure appropriate antibiotic use in hospitalized adults. Clin Infect Dis. 2015;60:281-91.

15. van Daalen FV, Prins JM, Opmeer BC, Boermeester MA, Visser CE, van Hest $\mathrm{RM}$, et al. A cluster randomized trial for the implementation of an antibiotic checklist based on validated quality indicators: the AB-checklist. BMC Infect Dis. 2015;15:134.

16. van Daalen FV, Prins JM, Opmeer BC, Boermeester MA, Visser CE, van Hest $\mathrm{RM}$, et al. Effect of an antibiotic checklist on length of hospital stay and appropriate antibiotic use in adult patients treated with intravenous antibiotics: a stepped wedge cluster randomized trial. Clin Microbiol Infect. 2017:23:485.e1-485.e8.

17. Thern J, de With K, Strauss R, Steib-Bauert M, Weber N, Kern WV. Selection of hospital antimicrobial prescribing quality indicators: a consensus among German antibiotic stewardship (ABS) networkers. Infection. 2014:42:351-62.

18. Schmitt C, Lacerda RA, Padoveze MC, Turrini RN. Applying validated quality indicators to surgical antibiotic prophylaxis in a Brazilian hospital: learning what should be learned. Am J Infect Control. 2012;40:960-2.

19. Kallen MC, Roos-Blom MJ, Dongelmans DA, Schouten JA, Gude WT, de Jonge $E$, et al. Development of actionable quality indicators and an action implementation toolbox for appropriate antibiotic use at intensive care units: A modified-rand Delphi study. PLOS ONE. 2018;13:e0207991.

20. Coenen S, Ferech M, Haaijer-Ruskamp FM, Butler CC, Vander Stichele RH, Verheij TJM, et al. European Surveillance of Antimicrobial Consumption
(ESAC): quality indicators for outpatient antibiotic use in Europe. Qual Saf Health Care. 2007;16:440-5.

21. Berrevoets MA, Ten Oever J, Sprong T, van Hest RM, Groothuis I, van Heij I, et al. Monitoring, documenting and reporting the quality of antibiotic use in the Netherlands: a pilot study to establish a national antimicrobial stewardship registry. BMC Infect Dis. 2017;17:565.

22. Arcenillas P, Boix-Palop L, Gómez L, Xercavins M, March P, Martinez L, et al. Assessment of quality indicators for appropriate antibiotic use. Antimicrob Agents Chemother. 2018;62:e00875-e918.

23. Adriaenssens N, Coenen S, Tonkin-Crine S, Verheij TJ, Little P, Goossens $H$, The ESAC Project Group. European Surveillance of Antimicrobial Consumption (ESAC): disease-specific quality indicators for outpatient antibiotic prescribing. BMJ Qual Saf. 2011;20:764-72.

24. Kallen MC, Prins JM. A systematic review of quality indicators for appropriate antibiotic use in hospitalized adult patients. Infect Dis Rep. 2017;9:6821.

25. Howard P, Huttner B, Beovic B, Beraud G, Kofteridis DP, Pano Pardo J, et al. ESGAP Indicators Working Group. ESGAP inventory of target indicators assessing antibiotic prescriptions: a cross-sectional survey. J Antimicrob Chemother. 2017;72:2910-4.

26. van den Bosch CM, Hulscher ME, Akkermans RP, Wille J, Geerlings SE, Prins JM. Appropriate antibiotic use reduces length of hospital stay. J Antimicrob Chemother. 2017;72:923-32.

27. Zarb P, Amadeo B, Muller A, Drapier N, Vankerckhoven V, Davey P, et al. Identification of targets for quality improvement in antimicrobial prescribing: the web-based ESAC Point Prevalence survey 2009. J Antimicrob Chemother. 2011:66:443-9.

28. van Daalen FV, Lagerburg A, de Kort J, Sànchez Rivas E, Geerlings SE. Implementation of an antibiotic checklist increased appropriate antibiotic use in the hospital on Aruba. Int J Infect Dis. 2017;59:14-21.

29. Gyssens IC. Quality measures of antimicrobial drug use. Int J Antimicrob Agents. 2001;17:9-19.

30. van den Bosch CM, Hulscher ME, Natsch S, Gyssens IC, Prins JM, et al. IEP. Development of quality indicators for antimicrobial treatment in adults with sepsis. BMC Infect Dis. 2014;14:345.

31. Centers for Disease Control and Prevention (CDC). The Core elements of hospital antibiotic stewardship program; 2019. Available at: https://www. cdc.gov/antibiotic-use/healthcare/pdfs/hospital-core-elements-H.pdf

32. Kim B, Lee MJ, Moon SM, Park SY, Song KH, Lee H, et al. Current status of antimicrobial stewardship programmes in Korean hospitals: results of a 2018 nationwide survey. J Hosp Infect. 2020;104:172-80.

\section{Publisher's Note}

Springer Nature remains neutral with regard to jurisdictional claims in published maps and institutional affiliations.
Ready to submit your research? Choose BMC and benefit from:

- fast, convenient online submission

- thorough peer review by experienced researchers in your field

- rapid publication on acceptance

- support for research data, including large and complex data types

- gold Open Access which fosters wider collaboration and increased citations

- maximum visibility for your research: over 100M website views per year

At BMC, research is always in progress.

Learn more biomedcentral.com/submissions 